\title{
ANÁLISIS DEL POLIMORFISMO CYP2C19*2 EN LA POBLACIÓN CASTELLANOLEONESA
}

\section{Analysis of polymorphism CYP2C19*2 in Castilla y León population}

Álvaro VELA; Carmen GUERRERO

Centro de Investigación del Cáncer, IBMCC, CSIC-USAL. Departamento de Medicina. Universidad de Salamanca, Salamanca, España

Autor de correspondencia: Carmen Guerrero. Centro de Investigación del Cáncer. Campus Unamuno, s/n. 37007-Salamanca. España

Correo-e: cguerrero@usal.es

RESUMEN: Las enzimas citocromo CYP P450 llevan a cabo el $75 \%$ de las reacciones de biotransformación de fármacos, por lo que sus polimorfismos tienen gran relevancia farmacocinética. Los polimorfismos del gen CYP2C19 afectan al metabolismo de fármacos antiulcerosos, inhibidores de la bomba de protones. Concretamente, el polimorfismo g.19154G>A da lugar a una función enzimática nula, lo que se traduce en una deficiente metabolización. Dicho polimorfismo afecta a un sitio de reconocimiento de la enzima de restricción Sma I, por lo que su detección se puede realizar mediante sencillas técnicas de biología molecular. Curiosamente, la presencia del polimorfismo se traduce en una mayor eficacia del tratamiento de erradicación de Helicobacter pilori. En este trabajo hemos analizado la incidencia de este polimorfismo en una muestra de 208 personas de la Comunidad Autónoma de Castilla y León, con énfasis en la población de Salamanca. Las proporciones globales de heterocigotos y homocigotos para el polimorfismo se ajustan a la literatura. Sin embargo, hemos encontrado un menor porcentaje de alelos polimórficos entre los individuos nacidos en Salamanca, en comparación con otras provincias. Sería interesante aumentar

Ediciones Universidad de Salamanca / @@ఠ $\quad$ FarmaJournal, vol. 5, núm. 1 (2020), pp. 95-104 
la muestra para esta población y estudiar la correlación de este polimorfismo con la alta incidencia de cáncer gástrico en la Comunidad.

Palabras clave: CYP2C19; polimorfismo; Helicobacter pilori; Cancer gástrico; Castilla y León.

ABSTRACT: Cytochrome CYP P450 enzymes carry out $75 \%$ of biotransformation reactions of drugs, so their polymorphisms have great pharmacokinetic relevance. The polymorphisms of the CYP2C19 gene affect the metabolism of antiulcer drugs, proton pump inhibitors. Specifically, the g.19154G > A polymorphism gives rise to a null enzyme, which results in poor metabolic activity. This polymorphism affects a recognition site for the restriction enzyme Sma I, so its detection can be carried out by simple molecular biology techniques. Interestingly, the presence of the polymorphism results in a greater efficacy of the Helicobacter pylori eradication treatment. In this work we have analyzed the incidence of this polymorphism in a sample of 208 people from the Autonomous Community of Castilla y León, with emphasis on the population of Salamanca. The global proportions of heterozygotes and homozygotes for the polymorphism are in agreement with the literature. However, we have found a lower percentage of polymorphic alleles among individuals born in Salamanca, compared to other provinces. It would be interesting to increase the sample for this population and study the correlation of this polymorphism with the high incidence of gastric cancer in the Community.

Keywords: CYP2C19; polymorpohism; Helicobacter pilori; Gastric cancer; Castilla y León.

\section{INTRODUCCIÓN}

Los polimorfismos son variaciones en la secuencia de nucleótidos del genoma humano que ocurren en al menos un 1\% de la población, lo que les diferencia de las mutaciones, mucho menos frecuentes. Las variaciones en su frecuencia están ligadas a condicionamientos étnicos y raciales, suponiendo desde un inofensivo cambio fenotípico hasta el incremento de la susceptibilidad a padecer una patología. Los polimorfismos de un único nucleótido o single nucleotide polymorphisms (SNP o snips), suponen más del 90\% de las variaciones del genoma humano, siendo los más frecuentes. Pueden darse en regiones codificantes, no codificantes o reguladoras, sin implicar siempre un cambio de aminoácido, debido a la degeneración del código genético. Los SNP a menudo introducen o eliminan sitios de reconocimiento de 
enzimas de restricción en regiones de DNA. Esto se traduce en una variabilidad en la longitud de los fragmentos generados tras la digestión del DNA con dichas enzimas. Este tipo particular de SNP se conoce como Polimorfismo de Longitud de Fragmentos de Restricción o RFLP (restriction fragment length polymorphism) (Rose et al., 2003).

La naturaleza polimórfica de los genes citocromo CYP P450, responsables del $75 \%$ de las reacciones de metabolización de fármacos, afecta a la respuesta interindividual de estos en clínica (Ingelman-Sundberg, Sim, 2010). Concretamente, el gen CYP2C19, presenta hasta 26 alelos funcionales diferentes (Ingelman-Sundberg, Sim, 2010). CYP2C19 es la enzima citocromo responsable del metabolismo de fármacos antiulcerosos, inhibidores de la bomba de protones (IBP) (IngelmanSundberg et al., 2007). El polimorfismo c.681G>A (g.19154G>A), situado en el exón 5 de CYP2C19, afecta a un codón de glicina (GGA) que se transforma en un sitio de procesamiento prematuro (GAA), lo que genera una proteína no funcional. Además, este cambio altera un sitio de reconocimiento de la enzima Sma I (CCCGGG), ya que el cambio afecta a la última base de esta secuencia, que pasaría a ser CCCGGA (Ingelman-Sundberg, Sim, 2010; Ozdil et al., 2010) (Figura 1A). Dependiendo de si el polimorfismo afecta a uno o a los dos alelos, podemos tener tres genotipos diferentes: homocigoto silvestre $(* 1 / * 1)$, heterocigoto para el polimorfismo $(* 1 / * 2)$ y homocigoto para el polimorfismo $(* 2 / * 2)$, cada uno con características fenotípicas particulares (El Rouby et al., 2018; Ingelman-Sundberg, Sim 2010; Tang et al., 2013). Así, la enzima silvestre (*1/*1), con una actividad enzimática normal, está considerada un metabolizador extensivo (EM, extensive metabolizer), mientras que las variantes con un alelo polimórfico $(* 1 / * 2)$ o los dos $(* 2 / * 2)$ son metabolizadores intermedio (IM, intermediate metabolizer) y lento (PM, poor metabolizer) respectivamente, pues el alelo polimórfico no es funcional.

Helicobacter pylori es una bacteria gramnegativa exclusiva del epitelio gástrico humano. Esta bacteria es la causante de úlceras estomacales y de gastritis, y está considerada el mayor factor de riesgo de cáncer gástrico (Pormohammad et al., 2019; Rokkas et al., 2017; Sugano, 2019). La infección por H. pylori se trata con una terapia triple consistente en dos antibióticos (normalmente amoxicilina y claritromicina) y un IBP como omeprazol, por lo que la eficacia del tratamiento está condicionada a los polimorfismos en CYP2C19 (Tang et al., 2013).

La variante alélica CYP2C19*2, en europeos, se encuentra en heterocigosis en un $20-35 \%$ de la población y en homocigosis en un 1-5\% de la población (El Rouby et al., 2018; Kuo et al., 2014). La tasa de éxito de la terapia de erradicación de Helicobacter pylori en úlceras gástricas está influenciada notablemente por el genotipo de CYP2C19. Se ha descrito que la tasa de erradicación es baja en el caso de metabolizadores extensivos, mientras que se ve incrementada considerablemente en metabolizadores intermedios y metabolizadores lentos (Kuo et al., 2014; Ozdil et al., 2010; Tang et al., 2013). 
El cáncer gástrico tiene una heterogénea distribución en España, siendo la Comunidad de Castilla y León la de mayor incidencia (Aragones et al., 2009). La presencia de Helicobacter pylori, subsecuente a la aparición de úlceras gástricas, junto con el elevado consumo de productos cárnicos en la Comunidad de Castilla y León, podría ser un factor relevante en la mayor incidencia de cáncer gástrico en su población (Zhao et al., 2017; Zhu et al., 2013).

El objetivo de este estudio es el análisis en una pequeña muestra de la población de Castilla y León, con énfasis en la población de Salamanca, de la incidencia del polimorfismo CYP2C19*2 en base a su relevancia por su posible conexión con la alta incidencia de cáncer gástrico en la comunidad.

\section{Materiales y métodos}

\subsection{Obtención de las muestras}

Se tomaron muestras de células epiteliales de la cavidad bucal a 217 voluntarios mayores de edad, con independencia de sexo y edad, nacidos en la Comunidad Autónoma de Castilla y León. Las muestras fueron obtenidas en Salamanca, entre julio y septiembre de 2017. Todos los voluntarios otorgaron su consentimiento verbal de participación en el estudio, el cual ha sido aprobado por el Comité Ético de Investigación del Hospital Universitario de Salamanca.

\subsection{Extracción del DNA del epitelio bucal}

Las células epiteliales se recogieron mediante frotado de una torunda estéril por la cavidad bucal, con énfasis en la zona de encías e interior de los carrillos, de forma que cada muestra de células fuera consistente. Para la extracción del DNA genómico se utilizó el CANVAX BS Buccal Swab Genomic DNA Extraction Kit, siguiendo las instrucciones del fabricante. Brevemente, se depositó la muestra en un tubo eppendorf conteniendo $750 \mu$ de solución de resuspensión. Tras centrifugado a $13000 \mathrm{rpm}$ durante $5 \mathrm{~min}$, se eliminó el sobrenadante dejando unos 10-20 $\mu \mathrm{l}$ de líquido residual. Las células se lisaron con una mezcla de 200 $\mu l$ de tampón de lisis S2 y $3 \mu$ le proteinasa $\mathrm{K}$, mediante incubación en un baño termostatizado a $56{ }^{\circ} \mathrm{C}$ durante $45-60 \mathrm{~min}$. Tras la precipitación y eliminación de las proteínas mediante centrifugación a $13000 \mathrm{rpm}$, el sobrenadante resultante fue transferido a un nuevo eppendorf conteniendo $190 \mu$ le isopropanol y 10 1 l de tampón S4. La nueva mezcla se homogeneizó por inversión permitiendo la precipitación del DNA. El precipitado se lavó con $200 \mu \mathrm{l}$ de etanol al 70\% y se secó durante 5 minutos a temperatura ambiente. El DNA de cada muestra se conservó en $20 \mathrm{ml}$ de tampón EB.

Ediciones Universidad de Salamanca / @@ఠ $\quad$ FarmaJournal, vol. 5, núm. 1 (2020), pp. 95-104 


\subsection{PCR, digestión del DNA y electroforesis}

Para la amplificación por PCR se diseñaron dos oligonucleótidos que definen un amplicón de $300 \mathrm{pb}$ del gen humano CYP2C19 conteniendo el exón 5, ya que el sitio polimórfico a analizar se encuentra en ese exón, concretamente en la posición 681 del cDNA (GenBack Accession NG_008384). Las secuencias del oligonucleótido directo y reverso fueron respectivamente: 5'-CAACCAGAGCTTGGCATATTG-3' y 5'-CACAAATACGCAAGCAGTCAC-3'. Dichos oligonucleótidos anillan en los intrones 4 y 5 respectivamente (Figura 1B). La

FIgURA 1. El polimorfismo en c.681G $>$ A del gen CYP2C19 afecta a un sitio de reconocimiento de la enzima de restricción Sma I

A
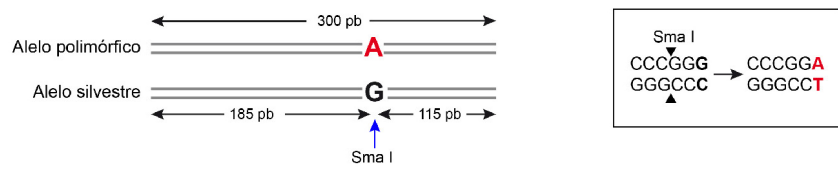

B

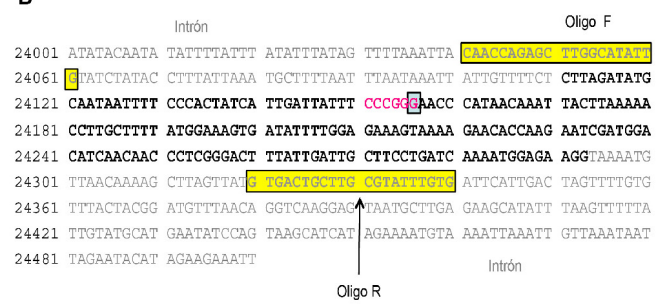

C

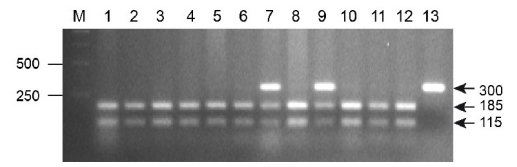

(A) Se indica la secuencia de reconocimiento, así como el punto de corte, y la localización de polimorfismo dentro de la secuencia. En ausencia de polimorfismo, Sma I divide el fragmento de $300 \mathrm{pb}$ en dos fragmentos de 185 y 115 pb. (B) Fragmento de la secuencia de CYP2C19 correspondiente al exón 5 y a fragmentos de los intrones flanqueantes donde anillan los oligonucleótidos utilizados en la reacción de PCR (cajas amarillas). Se indica en fucsia la secuencia de Sma I y con un recuadro el sitio polimórfico. Oligo F: oligo directo; oligo R: oligo reverso. (C) Ejemplo representativo de una electroforesis en gel de agarosa donde se analizan 13 de las muestras recogidas. Se observan las 3 bandas de 300 $\mathrm{pb}, 185 \mathrm{pb}$ y $115 \mathrm{pb}$. Las muestras 7 y 9 corresponden a genotipos heterocigóticos para el polimorfismo (GA); la muestra 13 corresponde a un genotipo homocigótico para el polimorfismo (AA). El resto de muestras presentan 2 bandas de $185 \mathrm{pb}$ y $115 \mathrm{pb}$, correspondientes al genotipo silvestre u homocigótico (GG). La figura representa concordancia con la distribución genotípica observada, siendo el silvestre (GG) el más frecuente y el homocigoto (AA), el menos habitual. M: Marcador de pesos moleculares (1 Kb ladder GeneRuler de Fisher Scientific). 
PCR se llevó a cabo a $55^{\circ} \mathrm{C}$ de anillamiento, 1 min, durante 35 ciclos. El producto de PCR se digirió con la enzima Sma I, 2 h a $30^{\circ} \mathrm{C}$. Los productos de digestión se visualizaron mediante electroforesis, en un gel de agarosa al 2\% (Figura 1C).

\section{Resultados}

De entre las 217 muestras analizadas, se obtuvieron 152 homocigotos silvestres o metabolizadores extensivos, 51 heterocigotos o metabolizadores intermedios y 5 homocigotos para el polimorfismo o metabolizadores lentos (Figura 2A). Las 9 muestras restantes fueron descartadas por la imposibilidad de obtener un resultado concluyente debido a la insuficiente cantidad de muestra recogida.

Figura 2. El polimorfismo en c.681G>A del gen CYP2C19 muestra una distribución diferencial en Castilla y León

A

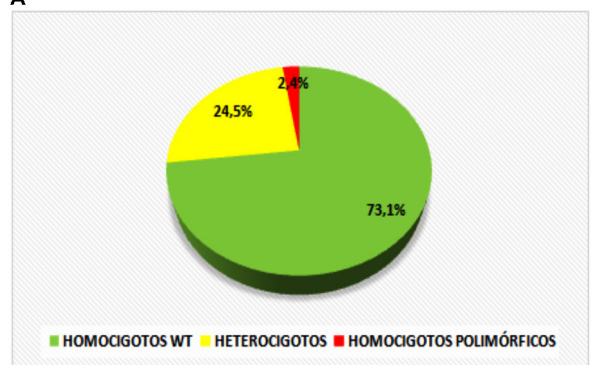

B

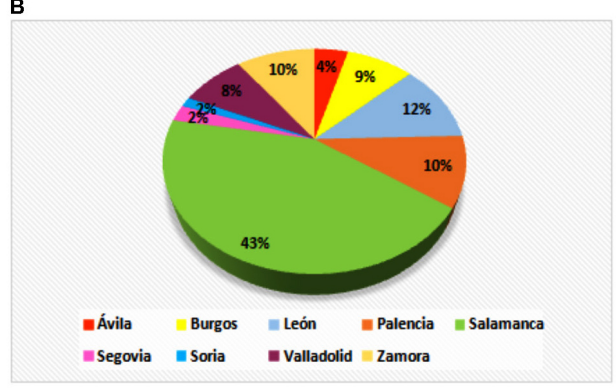
C

\begin{tabular}{lllll}
\hline PROVINCIAS & $\begin{array}{l}\text { N. } \\
\text { VÁLIDAS }\end{array}$ & $\begin{array}{l}\text { HOMOCIGOTOS } \\
\text { WT }\end{array}$ & HETEROCIGOTOS & $\begin{array}{l}\text { HOMOCIGOTOS } \\
\text { POLIMÓRFICOS }\end{array}$ \\
\hline Ávila & 9 & 4 & 4 & 1 \\
Burgos & 18 & 15 & 3 & 0 \\
León & 24 & 16 & 7 & 1 \\
Palencia & 21 & 13 & 7 & 1 \\
Salamanca & 90 & 71 & 18 & 1 \\
Segovia & 5 & 4 & 1 & 0 \\
Soria & 3 & 2 & 1 & 0 \\
Valladolid & 17 & 12 & 5 & 0 \\
Zamora & 21 & 15 & 5 & 1 \\
TOTAL & 208 & 152 & 51 & 5 \\
\hline
\end{tabular}

(A) Distribución genotípica obtenida de las 208 muestras válidas. El genotipo homocigótico silvestre fue el más frecuente, presentándose en un $73 \%$ de las muestras. El genotipo heterocigoto tuvo un porcentaje del $25 \%$ y el genotipo homocigoto para el polimorfismo solamente apareció en un 2\% de las muestras. (B) Distribución por provincias de las 208 muestras válidas recogidas. Se representa el porcentaje según el lugar de nacimiento de cada voluntario en las distintas provincias de la Comunidad Autónoma de Castilla y León. Como se aprecia, Salamanca es la provincia más representada. (C) Número de individuos de cada uno de los 3 genotipos por provincia de nacimiento.

Ediciones Universidad de Salamanca / @@ఠ $\quad$ FarmaJournal, vol. 5, núm. 1 (2020), pp. 95-104 
Para profundizar un poco más en el estudio, se analizaron estos mismos porcentajes por provincias. La Figura 2B muestra un gráfico con la distribución de las muestras recogidas según la provincia de nacimiento del donante. La mayor cantidad de muestras proceden de donantes de Salamanca, seguido de León, Palencia y Zamora, algo a tener en cuenta a la hora de analizar los datos. La Figura 2C muestra la distribución de todas las muestras por provincia y genotipo.

Finalmente, la Figura 3 recoge la distribución de los genotipos en las 4 provincias con mayor representación en número de muestras.

Figura 3. Distribución genotípica del polimorfismo en las 4 provincias donde se obtuvo un mayor número de muestras. Destaca la menor representación de homocigotos polimórficos en Salamanca, en comparación con las otras 3 provincias representadas
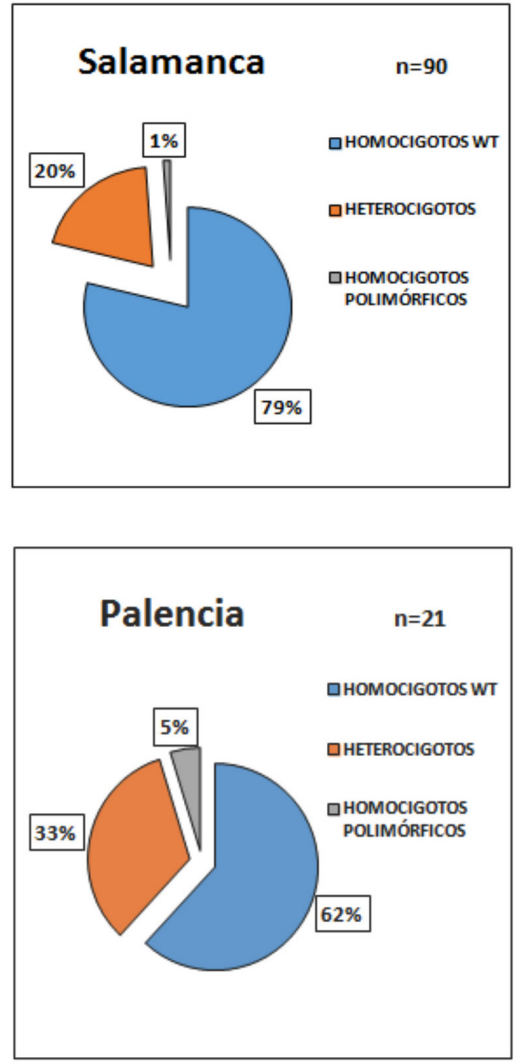

Ediciones Universidad de Salamanca / @@
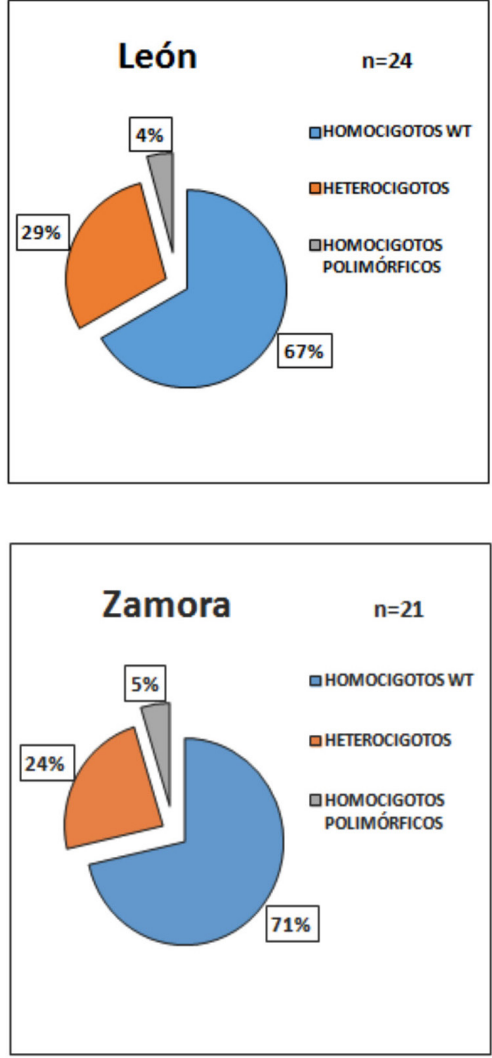

FarmaJournal, vol. 5, núm. 1 (2020), pp. 95-104 


\section{Discusión}

Los estudios farmacogenéticos están cada vez más asociados al desarrollo de fármacos con el objetivo de conseguir un tratamiento farmacológico más efectivo (Ingelman-Sundberg et al., 2007). El cribado de las variaciones polimórficas en enzimas como el citocromo CYP-P450, y concretamente en este caso CYP2C19, proporciona información acerca de cómo debe tratarse de forma individual a cada paciente según sus condicionamientos farmacogenéticos (Ingelman-Sundberg, Sim 2010). Para ello, conocer la frecuencia y repercusión del polimorfismo CYP2C19² en la población ayudará a adecuar las líneas de tratamiento con inhibidores de la bomba de protones (IBP) en la terapia de erradicación de Helicobacter pylori, con miras a mejorar su eficacia y seguridad de forma individualizada (Kuo et al., 2014; Tang et al., 2013).

Nuestros resultados han revelado una distribución genotípica del CYP2C19*2 en la población de Castilla y León, acorde a los datos conocidos hasta el momento (El Rouby et al., 2018; Kuo et al., 2014). Así, el genotipo hom(GG) silvestre EM es el más predominante con un $60-80 \%$ de los casos. Entre un $20-35 \%$ son het(GA) IM y solamente 1-5\% de la población presenta el polimorfismo en ambos alelos, es decir, son PM.

Si bien las frecuencias globales se ajustan a la literatura (El Rouby et al., 2018; Kuo et al., 2014), nos llama la atención la variabilidad entre provincias, sobre todo las diferencias entre Salamanca y las otras 3 provincias con un número representativo de muestras. Por ejemplo, de las 90 muestras de Salamanca solo hay 1 homocigoto polimórfico. Esto representa un 1,1\% frente al 5\% de este genotipo en Palencia y Zamora ( 1 caso en 21 muestras) y el 4\% en León (1 caso en 24 muestras). Sería interesante realizar estudios de asociación de este polimorfismo con la incidencia de cáncer gástrico en las distintas comunidades.

La influencia del genotipo CYP2C19*2 en el tratamiento de erradicación de Helicobacter pylori tiene relevancia, tanto en las primeras líneas de tratamiento, como en los tratamientos de rescate y el tratamiento con levofloxacino. La metabolización enzimática de IBP como omeprazol o lansoprazol por CYP2C19 va a determinar los niveles séricos de fármaco que llegan a su lugar de acción y la consiguiente efectividad del tratamiento. Se ha visto que en los EM la tasa de efectividad con la triple terapia de amoxicilina-claritromicina junto a un IBP se reduce un 15-25\% con respecto a los IM y PM (El Rouby et al., 2018; Kuo et al., 2014; Ozdil et al., 2010; Tang et al., 2013). Lo mismo ocurre en el caso de pantoprazol (El Rouby et al., 2018). Sin embargo, la presencia del CYP2C19*2 no afecta al tratamiento cuando el IBP incluido es rabeprazol o esomeprazol. Tampoco hay diferencias significativas con la triple terapia de rescate con levofloxacino (Kuo et al., 2014).

Ediciones Universidad de Salamanca / @@ఠ $\quad$ FarmaJournal, vol. 5, núm. 1 (2020), pp. 95-104 
A razón de esto, la eficacia terapéutica del tratamiento de erradicación de Helicobacter pylori en todos aquellos con presentación fenotípica de EM, se verá rebajada notablemente. Como hipotéticas medidas para obtener una terapia efectiva en toda la población se propone un aumento de dosis de omeprazol y lansoprazol, o el intercambio terapéutico por rabeprazol o esomeprazol (El Rouby et al., 2018; Tang et al., 2013). Además, la inserción de procedimientos de genotipado a los pacientes, en busca de los alelos polimórficos más comunes, podría suponer una reducción de costes en comparación con el uso de segundas y terceras líneas de tratamiento más costosas en aquellos pacientes cuyo tratamiento fracase en primer lugar (El Rouby et al., 2018).

Por último, la relación entre la infección de Helicobacter pylori y el elevado consumo de carne roja y productos cárnicos procesados en la comunidad de Castilla y León, son factores de riesgo que podrían incrementar la posibilidad de padecer cáncer gástrico (Zhao et al., 2017; Zhu et al., 2013). Sin embargo, se necesitan más estudios epidemiológicos que puedan evidenciar la influencia real de ambos factores, en conjunto, en el aumento de la incidencia de esta patología con respecto a otras comunidades (Aragones et al., 2009).

En resumen, las proporciones globales obtenidas en la población de Castilla y León, con énfasis en la población de Salamanca, del polimorfismo CYP2C19*2 se asemejan a las revisadas en la literatura para personas de procedencia europea. Por otra parte, a pesar de encontrarse entre los márgenes descritos, llama la atención el menor porcentaje de alelos polimórficos en los individuos nacidos en Salamanca, en comparación con otras provincias de la Comunidad, como Palencia, León o Zamora. Esta variabilidad entre diferentes provincias aún debe ser motivo de estudio, dado el pequeño tamaño de la muestra en la mayoría de las provincias.

\section{Bibliografía}

Aragones N, Pérez-Gómez B, Pollan M, Ramis R, Vidal E, Lope V, et al., The striking geographical pattern of gastric cancer mortality in Spain: environmental hypotheses revisited. BMC Cancer. 2019; 9:316.

El Rouby N, Lima JJ, Johnson JA. Proton pump inhibitors: from CYP2C19 pharmacogenetics to precision medicine. Expert Opin Drug Metab Toxicol. 2018; 14: 447-460.

Ingelman-Sundberg M, Sim SC (2010) Pharmacogenetic biomarkers as tools for improved drug therapy; emphasis on the cytochrome P450 system. Biochem Biophys Res Commun. 2018; 396:90-94.

Ingelman-Sundberg M, Sim SC, Gómez A, Rodríguez-Antona C. Influence of cytochrome P450 polymorphisms on drug therapies: pharmacogenetic, pharmacoepigenetic and clinical aspects. Pharmacol Ther. 2007; 116:496-526. 
Kuo CH, Lu CY, Shih HY, Liu CJ, Wu MC, Hu HM, et al., CYP2C19 polymorphism influences Helicobacter pylori eradication. World J Gastroenterol. 2014; 20:1602916036.

Ozdil B, Akkiz H, Bayram S, Bekar A, Akgollu E, Sandikci M. Influence of CYP2C19 functional polymorphism on Helicobacter pylori eradication. Turk J Gastroenterol. 2010; 21:23-28.

Pormohammad A, Mohtavinejad N, Gholizadeh P, Dabiri H, Salimi Chirani A, Hashemi A, et al., Global estimate of gastric cancer in Helicobacter pylori-infected population: A systematic review and meta-analysis. J Cell Physiol. 2019; 234:1208-1218.

Rokkas T, Rokka A, Portincasa P. A systematic review and meta-analysis of the role of Helicobacter pylori eradication in preventing gastric cancer. Ann Gastroenterol. 2017; 30:414-423.

Rose CM, Marsh S, Ameyaw MM, McLeod HL. Pharmacogenetic analysis of clinically relevant genetic polymorphisms. Methods Mol Med. 2003; 85:225-237.

Sugano K. Effect of Helicobacter pylori eradication on the incidence of gastric cancer: a systematic review and meta-analysis. Gastric Cancer. 2019; 22:435-445.

Tang HL, Li Y, Hu YF, Xie HG, Zhai SD. Effects of CYP2C19 loss-of-function variants on the eradication of $\mathrm{H}$. pylori infection in patients treated with proton pump inhibitor-based triple therapy regimens: a meta-analysis of randomized clinical trials. PLoS One. 2013; 8:e62162.

Zhao Z, Yin Z, Zhao Q. Red and processed meat consumption and gastric cancer risk: a systematic review and meta-analysis. Oncotarget. 2017; 8:30563-30575.

Zhu H, Yang X, Zhang C, Zhu C, Tao G, Zhao L, et al., Red and processed meat intake is associated with higher gastric cancer risk: a meta-analysis of epidemiological observational studies. PLoS One. 2013; 8:e70955. 